\title{
Revisiting Composite Right-/Left-Handed Transmission Lines and Couplers
}

\author{
B. Honarbakhsh
}

\begin{abstract}
In this paper, some hidden aspects of composite right-/left-handed (CRLH) transmission lines (TLs) and couplers are clarified. Complete and detailed dispersion analysis of an isolated CRLH TL is presented including lossless and lossy cases on a conventional unit cell, devised formerly. The left-handed behavior of such TLs is confirmed. Dispersion analysis of CRLH TLs is extended form the conventional to an accurate unit cell. Capability of TLs consisting of such unit cells in supporting RLH waves is demonstrated. A pure analytical strategy is proposed for parameter extraction of CRLH TLs and symmetric couplers based on accurate circuit models.
\end{abstract} line.

Index Terms - coupler, left-handed, metamaterial, transmission

\section{INTRODUCTION}

$\mathrm{T}$ HE purpose of this paper is to clarify some hidden aspects of a rather old but still attractive transmission line (TL) approach to left-handed metamaterials. This valuable approach, introduced in 2003 as a conference paper [1] and then developed and presented as a book in 2006 [2], has become a powerful engineering tool for design of advantageous microwave components [3]-[13].

Two main issues are revisited; i.e., dispersion analysis of a composite right-/left-handed (CRLH) TL and parameter extraction of both CRLH TLs and symmetric edge couplers. Dispersion analysis of lossless CRLH TL is formerly carried out in [2], [14]. For the lossless case, it is not rigorously shown how the correct branch of the phase constant can be chosen. For the lossy TL, the analysis is restricted to the low-loss case. In [2] and [3], parameter extraction is performed for a CRLH TL and a symmetric CRLH coupler, respectively. For both of these structures, only the lossless case is considered. Additionally, in contrast to an isolated CRLH TL, the suggested strategy for extracting circuit parameters of the coupler requires curve fitting methods and thus is not purely analytic [2],[3], [9]. This violates the purpose of the circuit model since curve fitting is potentially a time-consuming process and may lead to losing the correct phase response of the corresponding scattering (S-) parameters. The circuit model not only provides an intuitive description of the physical model but also, serves as a valuable design tool. Thus, its construction should take less time than a full-wave analysis of the complete structure. The said deficiencies are resolved in the present work. Specifically, in section 2, it is shown that for a lossless CRLH TL, it is not possible to uniquely determine the phase constant. The situation is similar to the uniqueness theorem in electromagnetics that cannot be proven in a lossless media [15]. Then, a complete dispersion analysis of a lossy CRLH TL is provided and the complex propagation constant is uniquely determined. Besides, it is shown that the lossless TL can be well described as the limiting case of the lossy TL. In section 3, a general strategy is suggested to obtain dispersion curves of a CRLH TL using an accurate circuit model of the corresponding unit cell. In section 4 , a method for parameter extraction of a CRLH TL is proposed based on the said unit cell. Finally, section 5 is devoted to detailed parameter extraction of symmetric CRLH edge coupler solely based on analytic expressions. Through the paper, the unit cell of all physical CRLH TLs is the same as the one proposed in [3] that is consisted of cascading an interdigital capacitor (IDC) and a short-circuited stub inductor (SI). The full-wave simulations are carried out using Keysight ${ }^{\circledR}$ Momentum.

\section{REVISITING DiSPERSION ANALYSIS}

The unit cell of a conventional CRLH TL is depicted in Fig. 1, wherein $Z_{s}, Y_{p}$, and $\delta$ are, respectively, series impedance, parallel admittance and physical length [2]. The complex propagation constant of a TL consisted from cascading infinite number of such a unit cell can be represented by [16]:

$$
\gamma=\lim _{\delta \rightarrow 0} \frac{1}{\delta^{2}} \sqrt{Z_{s} Y_{p}}
$$

that can also be regarded as the dispersion relation. Subsequently, real and imaginary parts of the propagation constant are derived as a function of angular frequency for

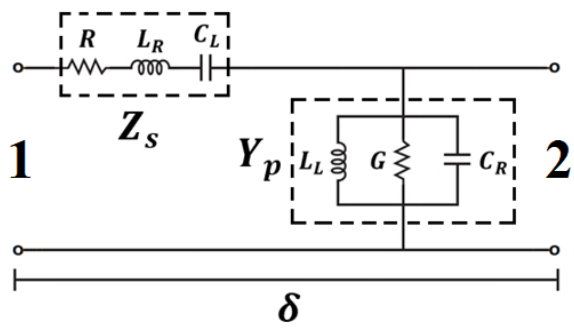

Fig. 1. The unit cell of a conventional CRLH TL.
Babak Honarbakhsh is with the Faculty of Electrical Engineering, Shahid Beheshti University, 1983963113, Tehran, Iran (e-mail: b_honarbakhsh@sbu.ac.ir). 
lossless and lossy TLs. Note that due to the conservation of energy, the real part of the propagation constant must be nonnegative. All derivations of this section are at the level of highschool mathematics and thus only the final results are included. Besides, following [2],

$$
\begin{gathered}
\omega_{s e}=1 / \sqrt{L_{R} C_{L}}, \omega_{s h}=1 / \sqrt{L_{L} C_{R}} \\
\omega_{\min }=\min \left(\omega_{s e}, \omega_{s h}\right), \omega_{\max }=\max \left(\omega_{s e}, \omega_{s h}\right)
\end{gathered}
$$

\section{A. Lossless CRLH TL}

In this case, the series impedance and parallel admittance are:

$$
Z_{s, l l}=j \omega L_{R}+1 /\left(j \omega C_{L}\right), Y_{p, l l}=j \omega C_{R}+1 /\left(j \omega L_{L}\right)
$$

and the dispersion equation is:

$$
\gamma_{l l}^{2}=\left(\alpha_{l l}+j \beta_{l l}\right)^{2}=f_{1}(\omega)
$$

wherein the subscript "ll" stands for lossless. Also, according to [2],

$$
\begin{aligned}
f_{1} & =-\left[\left(\omega / \omega_{R}\right)^{2}+\left(\omega_{L} / \omega\right)^{2}+\kappa \omega_{L}^{2}\right] \\
& >0, \quad \omega_{\min }<\omega<\omega_{\max } \\
& <0, \quad \text { o.w. }
\end{aligned}
$$

Since $\alpha_{l l} \geq 0$ and $\beta_{l l} \in \mathbb{R}$, it can be concluded that:

$$
\alpha_{l l}= \begin{cases}+\sqrt{\left|f_{1}\right|}, & \omega_{\min }<\omega<\omega_{\max } \\ 0, & \text { o.w. }\end{cases}
$$

and

$$
\beta_{l l}= \begin{cases}0, & \omega_{\min }<\omega<\omega_{\max } \\ \pm \sqrt{\left|f_{1}\right|}, & \text { o.w. }\end{cases}
$$

which defines two pass-bands and one stop-band. Note that in the stop-band, the amplitude of the voltage wave is non-zero. Though, its gradient is zero due to the zero value of the phase constant. Besides, $\beta_{l l}$ cannot be uniquely determined in passbands. The characteristic impedance of the TL is:

$$
\begin{aligned}
Z_{c, l l} & =\sqrt{Z_{s, l l} / Y_{p, l l}}=Z_{R} \sqrt{\frac{\omega^{2}-\omega_{s e}^{2}}{\omega^{2}-\omega_{s h}^{2}}} \\
& \in \mathbb{I}, \quad \omega_{\min }<\omega<\omega_{\max } \\
& \in \mathbb{R}^{+}, \quad \text { o.w. }
\end{aligned}
$$

and thus, the corresponding reflection coefficient with respect to a real characteristic impedance is:

$$
\begin{aligned}
\mathrm{S}_{11}(\mathrm{~dB}) & =0, \quad \omega_{\min }<\omega<\omega_{\max } \\
& <0, \quad \text { o.w. }
\end{aligned}
$$

This shows complete reflection at the stop-band. Note that at the stop-band, there is no thermodynamic loss. Also, the attenuation constant is finite.

\section{B. Lossy CRLH TL}

In this case, the series impedance and parallel admittance are:

$$
Z_{s, l}=R+Z_{s, l l}, \quad Y_{p, l}=G+Y_{p, l l}
$$

and the dispersion equation is:

$$
\gamma_{l}^{2}=\left(\alpha_{l}+j \beta_{l}\right)^{2}=f_{2}(\omega)+j f_{3}(\omega)
$$

wherein the subscript " $l$ " stands for lossy and

$$
\begin{gathered}
f_{2}=R G+f_{1} \\
f_{3}=R C_{R}\left(\omega-\omega_{s h}^{2} / \omega\right)+G L_{R}\left(\omega-\omega_{s e}^{2} / \omega\right)
\end{gathered}
$$

By defining $\Delta$ and $\omega_{b}$ as

$$
\begin{aligned}
\Delta=\left[\left(\omega_{s e}+\omega_{s h}\right)^{2}-R G \omega_{R}^{2}\right]\left[\left(\omega_{s e}-\omega_{s h}\right)^{2}-R G \omega_{R}^{2}\right] \\
\omega_{b}=\sqrt{\left(R C_{R} \omega_{s h}^{2}+G L_{R} \omega_{s e}^{2}\right) /\left(R C_{R}+G L_{R}\right)}
\end{aligned}
$$

it can be shown that:

$$
\begin{aligned}
& f_{2}<0, \Delta<0, \quad \forall \omega \\
& \quad>0, \Delta \geq 0, \quad \omega_{1}<\omega<\omega_{2} \\
& \quad<0, \text { o.w. }
\end{aligned}
$$

and

$$
f_{3} \gtrless 0, \omega \gtrless \omega_{b}
$$

where

$$
\omega_{1,2}=\frac{\sqrt{2}}{2} \sqrt{\left[\left(\omega_{s e}^{2}+\omega_{s h}^{2}\right)-R G \omega_{R}^{2}\right] \mp \sqrt{\Delta}}
$$

Additionally,

$$
\begin{gathered}
\Delta<0 \Leftrightarrow\left|\sqrt{\frac{L_{R}}{L_{L}}}-\sqrt{\frac{C_{R}}{C_{L}}}\right|<\sqrt{R G}<\left|\sqrt{\frac{L_{R}}{L_{L}}}+\sqrt{\frac{C_{R}}{C_{L}}}\right| \\
\omega_{\min } \leq \omega_{b} \leq \omega_{\max }
\end{gathered}
$$

Since $\alpha_{l} \geq 0$ and $\beta_{l} \in \mathbb{R}$, solving (11) for $\alpha_{l}$ and $\beta_{l}$ leads to:

$$
\alpha_{l}=\frac{\sqrt{2}}{2}\left(f_{2}+\sqrt{f_{2}^{2}+f_{3}^{2}}\right)>0, \quad \forall \omega
$$

and

$$
\beta_{l}= \begin{cases}-\frac{\sqrt{2}}{2} \sqrt{-f_{2}+\sqrt{f_{2}^{2}+f_{3}^{2}},} & \omega<\omega_{b} \\ +\frac{\sqrt{2}}{2} \sqrt{-f_{2}+\sqrt{f_{2}^{2}+f_{3}^{2}},} & \omega>\omega_{b}\end{cases}
$$

Thus, the line is LH for $\omega<\omega_{b}$ and is RH for $\omega>\omega_{b}$.

\section{Lossless CRLH TL as limiting case of lossy CRLH TL}

As a familiar technique, a lossless TL can be considered as the limiting case of the lossy TL when loss parameters approach 
zero. Note that the limit of $\omega_{b}$ does not exist when $(R, G) \rightarrow$ $(0,0)$ but $(17 \mathrm{~b})$ guarantees that its limiting value is bounded by $\omega_{\min }$ and $\omega_{\max }$. Thus, at the limit, the sign of $\beta_{l}$ cannot be determined when $\omega_{1}<\omega<\omega_{2}$. Also, (12) and (16) lead to:

$$
\begin{gathered}
\lim _{(R, G) \rightarrow(0,0)} f_{2}=f_{1,} \lim _{(R, G) \rightarrow(0,0)} f_{3}=0 \\
\lim _{(R, G) \rightarrow(0,0)} \omega_{1}=\omega_{\text {min }}{ }_{(R, G) \rightarrow(0,0)} \omega_{2}=\omega_{\max }
\end{gathered}
$$

Consequently,

$$
\begin{aligned}
\lim _{(R, G) \rightarrow(0,0)} \alpha_{l} & =\frac{\sqrt{2}}{2}\left(f_{1}+\left|f_{1}\right|\right), \quad \forall \omega \\
& =\sqrt{2}\left|f_{1}\right|, \quad \omega_{\min }<\omega<\omega_{\max } \\
& =0, \quad \text { o.w. }
\end{aligned}
$$

and

$$
\begin{aligned}
& \lim _{(R, G) \rightarrow(0,0)} \beta_{l}= \begin{cases}+\frac{\sqrt{2}}{2} \sqrt{-f_{1}+\left|f_{1}\right|}, & \omega>\omega_{b} \\
-\frac{\sqrt{2}}{2} \sqrt{-f_{1}+\left|f_{1}\right|}, & \omega<\omega_{b}\end{cases} \\
& =-\sqrt{2}\left|f_{1}\right|, \quad \omega<\omega_{\min } \\
& =0, \quad \omega_{\min }<\omega<\omega_{\max } \\
& =+\sqrt{2}\left|f_{1}\right|, \quad \omega>\omega_{\max }
\end{aligned}
$$

Therefore, in contrast to the lossless case, the phase constant of a lossy TL is uniquely determined. Note that in the stop-band, the sign of $\beta_{l}$ is unknown but its value is known.

\section{ReVISITING CRLH TL UNIT CELL}

A systematic method to design and analysis of a CRLH TL is decomposing its unit cell into two cascaded sub-cells; one to provide the series capacitance and the other, to provide the parallel inductance (Fig. 2) [2]. In almost all such designs, each of the sub-cells can be modeled by a symmetric $\Pi$ or T network. From the circuit theory standpoint, the order of such a combination is greater than that of the conventional CRLH unit cell and thus, those are not equivalent in the strict sense. However, the validity of such cells in providing CRLH behavior is certain. In this section, it is shown that desired equivalence holds when the cell size approaches zero. In so doing, the following steps were carried out using MATLAB ${ }^{\circledR}$ Symbolic Computation Toolbox. First, the $A B C D$-matrix of cascade combination of the T-networks corresponding to each sub-cell was computed.

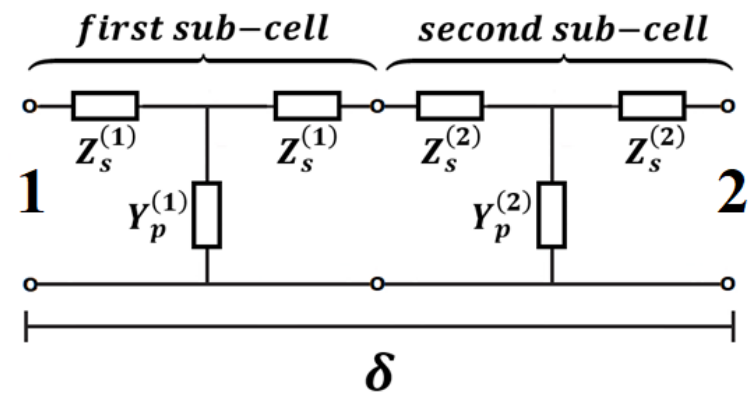

Fig. 2. The unit cell of a CRLH TL consisted of cascading of two sub-cells.
Second, by using the said matrix and applying periodic boundary conditions, the determinantal equation governing the corresponding uniform TL was developed [2]; i.e.,

$$
A D-(A+D) e^{\gamma \delta}+e^{2 \gamma \delta}-B C=0 .
$$

Third, the exponential functions in (23) were replaced by the first three terms of their Taylor's series expansion. Forth, the leading term of the resulting polynomial in $\delta$ was extracted which contains the desired dispersion equation. The derived dispersion relation exactly matches that of the conventional CRLH unit cell with:

$$
Z_{s}^{(e q)}=\sqrt{\frac{2}{3}}\left(Z_{s}^{(1)}+Z_{s}^{(2)}\right), Y_{p}^{(e q)}=\sqrt{\frac{2}{3}}\left(Y_{p}^{(1)}+Y_{p}^{(2)}\right)
$$

wherein the superscript "eq" stands for "equivalent" and

$$
\begin{gathered}
Z_{s}^{(1)}=R^{(1)}+j \omega L_{s}^{(1)}+1 /\left(j \omega C_{s}^{(1)}\right), Y_{p}^{(1)}=G^{(1)}+j \omega C_{p}^{(1)} \\
Z_{s}^{(2)}=R^{(2)}+j \omega L_{s}^{(2)}, Y_{p}^{(2)}=G^{(2)}+j \omega C_{p}^{(2)}+1 /\left(j \omega L_{p}^{(2)}\right) \\
\text { IV. REVISITING PARAMETER EXTRACTION OF } \\
\text { CRLH TRANSMISSION LINES }
\end{gathered}
$$

\section{REVISITING PARAMETER EXTRACTION OF CRLH TRANSMISSION LINES}

In [2], an analytic method is proposed for parameter extraction of CRLH TLs. The attained circuit model is beneficial from two aspects. First, it simplifies the design and analysis of such TLs. Second, dispersion analysis of the line becomes straightforward. Yet, three simplifications have been considered. First, the devised closed-form formulas are based on the lossless line. Second, the circuit elements are extracted at a single frequency. Third, the unit cell of the physical TL is forced to be equivalent to the lossless version of Fig. 1.

Especially, the last simplification is not possible in the strict sense as explained in section 3 . The benefits of the circuit model are preserved by including loss parameters without overloading of computational resources. Noting to the previous section, the accurate circuit model exactly matches its conventional counterpart at the limit. Thus, there is no need to enforce the conventional circuit to the physical model. As well, even when low-loss dielectrics are exploited in the physical realization of CRLH TLs, the values of lossy elements are not necessarily negligible. It is well-known that CRLH TLs are potentially good radiators are their application as antennas are justified long ago [2]. In such cases, including the series resistance in the circuit model is judicious to properly model the radiation loss. Also, performing dispersion analysis based on the accurate circuit model is straightforward. For this purpose, one can first transform (23) to a quadratic equation in variable $X$ with $X=e^{\gamma \delta}$ and then, since $\alpha_{l l} \geq 0$, select the desired solution such that $|X| \leq 1$. This procedure is both general and accurate. Another simplification that has been followed thus far, is restricting the parameter extraction to a single frequency. Fullwave analysis of a unit cell is not computationally costly and thus, frequency-dependent circuit parameters can be computed in the whole frequency band to provide an accurate description of the real structure. At last, providing closed-form formulas is 
simple for lossy TLs. Without loss of generality, consider Fig. 2 as the desired circuit model. The impedance matrices of subcells are:

$$
\mathbf{Z}^{(k)}=\left[\begin{array}{cc}
Z_{s}^{(k)}+1 / Y_{p}^{(k)} & 1 / Y_{p}^{(k)} \\
1 / Y_{p}^{(k)} & Z_{s}^{(k)}+1 / Y_{p}^{(k)}
\end{array}\right], k=1,2
$$

that can be obtained from full-wave simulation. Following the parameter extraction strategy proposed in [2], it can be shown that:

$$
\begin{gathered}
R^{(1)}=\operatorname{Re}\left\{Z_{s}^{(1)}\right\} \\
L_{s}^{(1)}=\frac{1}{j 2 \omega}\left(j \cdot \operatorname{Im}\left\{Z_{s}^{(1)}\right\}+\omega \frac{\partial Z_{s}^{(1)}}{\partial \omega}\right) \\
C_{s}^{(1)}=\frac{j \omega}{2}\left(j \cdot \operatorname{Im}\left\{Z_{s}^{(1)}\right\}-\omega \frac{\partial Z_{s}^{(1)}}{\partial \omega}\right)^{-1} \\
G^{(1)}=\operatorname{Re}\left\{Y_{p}^{(1)}\right\} \\
C_{p}^{(1)}=\frac{1}{\omega} \operatorname{Im}\left\{Y_{p}^{(1)}\right\}
\end{gathered}
$$

and

$$
\begin{gathered}
R^{(2)}=\operatorname{Re}\left\{Z_{s}^{(2)}\right\} \\
L_{s}^{(2)}=\frac{1}{\omega} \operatorname{Im}\left\{Z_{s}^{(2)}\right\} \\
G^{(2)}=\operatorname{Re}\left\{Y_{p}^{(2)}\right\} \\
C_{p}^{(2)}=\frac{1}{j 2 \omega}\left(j \cdot \operatorname{Im}\left\{Y_{p}^{(2)}\right\}+\omega \frac{\partial Y_{p}^{(2)}}{\partial \omega}\right) \\
L_{p}^{(2)}=\frac{j \omega}{2}\left(j \cdot \operatorname{Im}\left\{Y_{p}^{(2)}\right\}-\omega \frac{\partial Y_{p}^{(2)}}{\partial \omega}\right)^{-1}
\end{gathered}
$$

Other variants of the circuit model can be used wherein a Пnetwork is replaced for each sub-cell. The 9-cell CRLH TL presented in [2], [3] was analyzed with different methods to validate the claims of this section. The first and second sub-cells corresponds to, respectively, IDC a SI. The approximate circuit simulations are based on the parameter values reported in [2]. The accurate circuit simulations are based on (27) and (28). The attenuation constant and transmission characteristic is reported in Figs. 3 and 4, respectively, that clearly show the superiority of the accurate model over its conventional counterpart. A perfect match between full-wave and circuit simulations is impossible since mutual coupling between sub-cells of each unit cell and between different unit cells are not included in the circuit model. Note, also, that parameter extraction of each subcell must be carried out separately. All the attempts of the author to extract the circuit parameters from the combined unit cell failed. Probably, this is due to the resonance nature of the whole unit cell. It is suitable to modify all of the matrices describing the unit cell such that to satisfy symmetry and reciprocity. Although the real structure is both symmetric and reciprocal, the obtained network representation from the fullwave simulation may not exactly satisfy these properties due to numerical error. It is also useful to take the advantage of averaging, whenever possible. Say, replace $z_{11}$ and $z_{22}$ by $\left(z_{11}+z_{22}\right) / 2$. Similarly, replace $z_{12}$ and $z_{21}$ by $\left(z_{12}+z_{21}\right) / 2$.

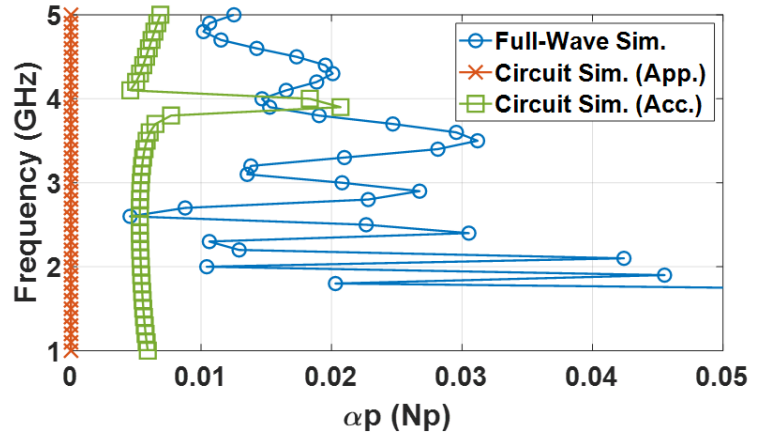

Fig. 3. Attenuation constant of a 9-cell CRLH TL designed in [2].

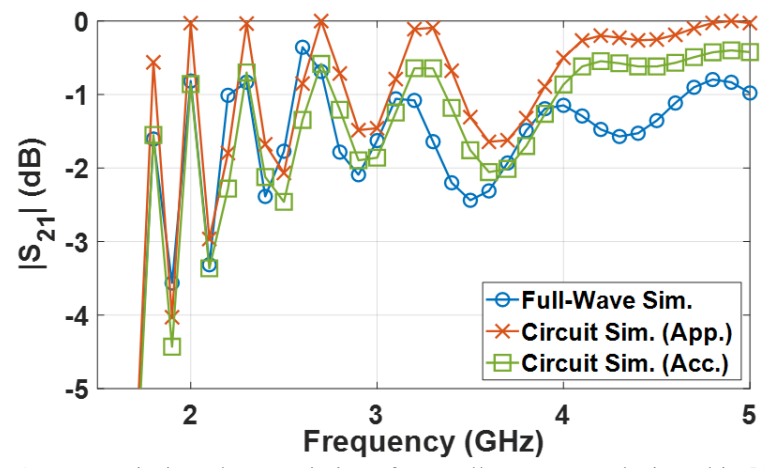

Fig. 4. Transmission characteristics of a 9-cell CRLH TL designed in [2].

\section{REVISITING PARAMETER EXTRACTION OF CRLH SYMMETRIC COUPLERS}

In [3], the theory of CRLH TLs has been extended to a symmetric edge coupler. A complete analysis is reported that is validated by both full-wave simulation and measurement. Yet, all of the simplifying assumptions pointed out in the previous section have been used for developing the circuit model. Besides, details of the parameter extraction are not included. Especially, coupling parameters are extracted using curve fitting. It is worth mentioning that switching to curve fitting violates the purpose of the circuit model due to two main reasons. First, it is generally time-consuming, and second, it may degrade the phase response. Note that the value of the circuit model for a coupler is much more compared to that of an isolated TL since the full-wave analysis of the former is significantly more time-consuming compared to that of the latter. As a result, easy access and an accurate circuit model can speed up the design and optimization of CRLH couplers.

In the present section, the said deficiencies are removed. The circuit model of the unit cell for the symmetric coupler is depicted in Fig. 5. It is well known that the systematic method for analysis of such structure is even-odd mode decomposition [16] by which it becomes possible to use analysis formulas of an isolated CRLH TL by properly modify the unit cell immittances [3]. Specifically, the circuit model of the TL corresponding to the even mode is the same as the one depicted in Fig. 2 wherein $L_{s}^{(k)}$ is replaced by $L_{s}^{(k)}+L_{m}^{(k)}$ for $k=1,2$. Similarly, the circuit model of the odd mode, is the same as the one depicted in Fig. 2 wherein $C_{p}^{(k)}$ is replaced by $C_{p}^{(k)}+C_{m}^{(k)}$ for $k=1,2$. Note that, compared to an isolated TL, one new parameter is added to each of the said even and odd TL circuit 
models; i.e., coupling inductors and coupling capacitors. Likely, the addition of the corresponding unknowns was the reason for using carve fitting methods for parameter extraction in [3]. By assuming that common parameters in even and odd modes have equal values, it is still possible to extract all circuit parameters, analytically. For instance, since the total series inductance of the first sub-cell in even and odd TLs is, respectively, $L_{s}^{(1)}+2 L_{m}^{(1)}$ and $L_{s}^{(1)}$, computing of $L_{m}^{(1)}$ is trivial assuming that $L_{s}^{(1)}$ in the even mode equals $L_{s}^{(1)}$ in the odd mode. To validate the said claim, extracted parameters of the sub-cells of the coupler with the unit cell proposed in [3] is reported in Figs. 6 and 7 wherein subscripts " $e$ " and " $o$ " stands for "even" and "odd", respectively. Similar to the case of isolated TL, the first and second sub-cells correspond to, respectively, IDC a SI. As can be seen, values of the reactive parameters are essentially the same in even and odd modes. Nevertheless, loss parameters differ significantly for different modes. Besides, although a low loss substrate has been used for microstrip realization of the coupler, huge values for loss parameters are obtained which is due to even-odd mode decomposition. Similar to the case of an isolated TL, parameters of each sub-cell are extracted separately. Also, symmetry and reciprocity conditions together with averaging are imposed on simulation results.

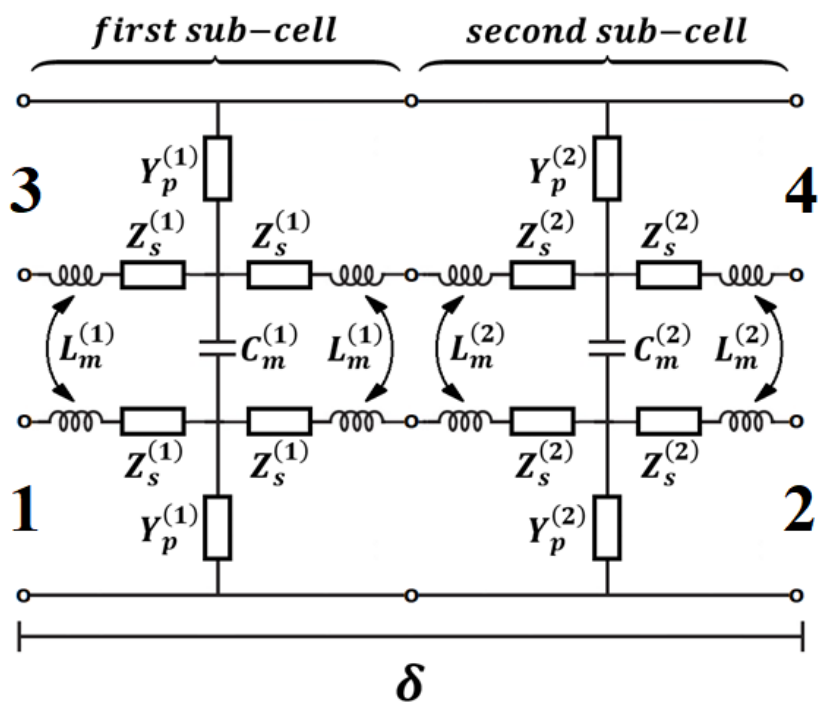

Fig. 5. Circuit model of the unit cell for the symmetric edge coupler.

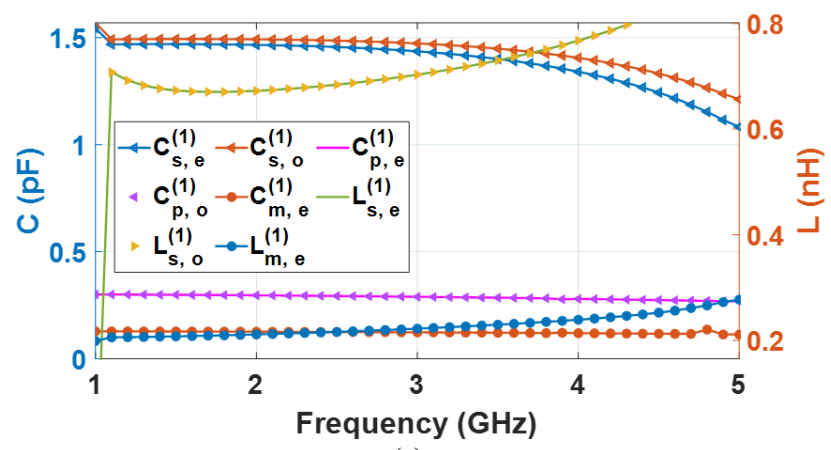

(a)

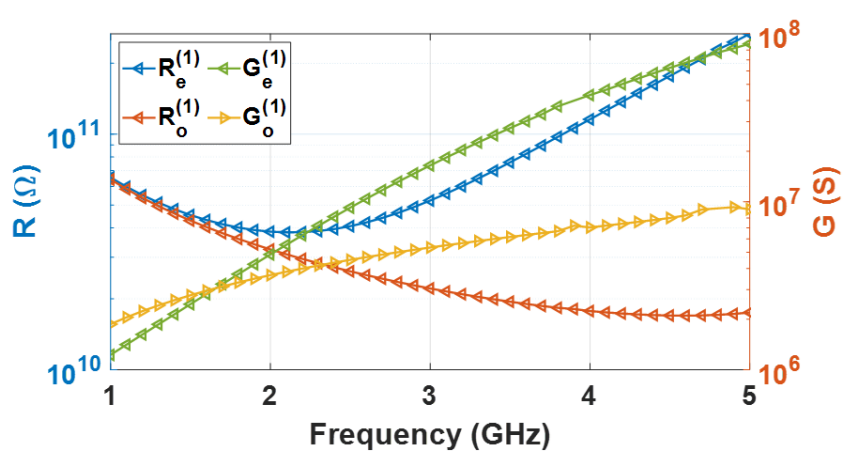

(b)

Fig. 6. Extracted parameters of the first sub-cell for even and mode TLs: (a) reactive elements, (b) lossy elements.

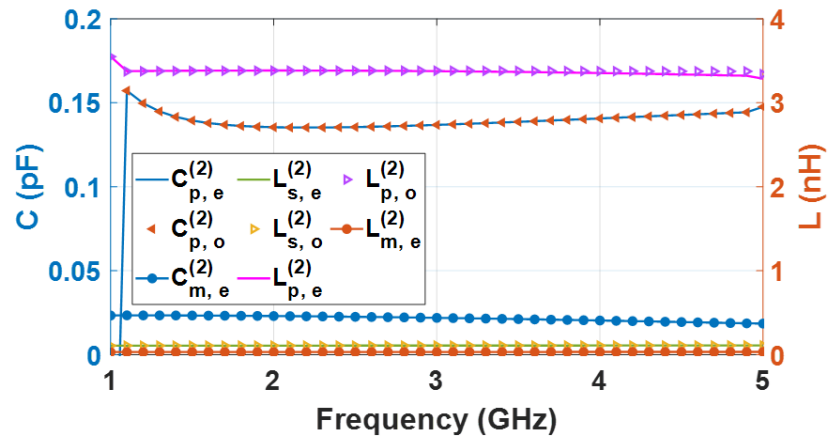

(a)

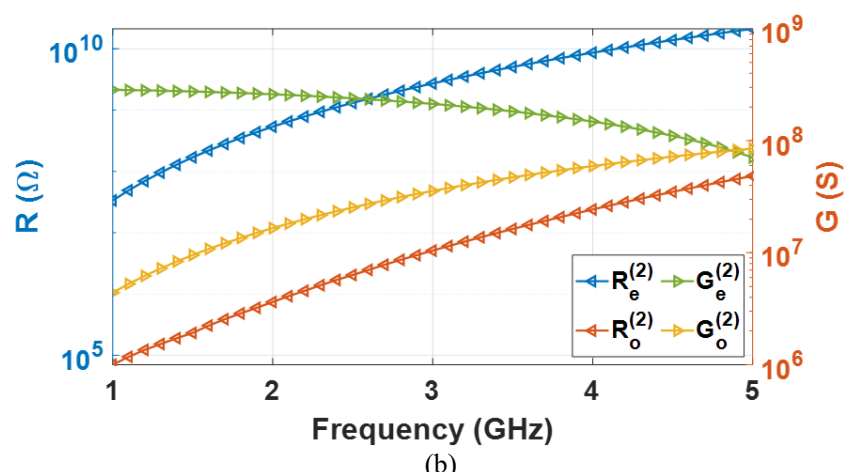

Fig. 7. Extracted parameters of the second sub-cell for even and odd modes: (a) reactive elements, (b) lossy elements.

S-parameters computed by full-wave and circuit simulations are reported in Fig. 8 that are in good agreement. Results of the circuit simulations reported in [3] are not included since the author was unable to regenerate them based on the reported model parameters (I think there are some typos in [3].). Note that expecting "excellent agreement" is not realistic due to various couplings between components of the unit cell which are not included in the circuit model; i.e., coupling between subcells of each branch of the coupler and cross-couplings between sub-cells of different branches. Also, note that the circuit parameters obtained in [3] have not led to such a good agreement. Specifically, two sets of S-parameters $\left(\left|\mathrm{S}_{21}\right|\right.$ and $\left.\left|S_{31}\right|\right)$ are in very good agreement. However, two other sets $\left(\left|\mathrm{S}_{11}\right|\right.$ and $\left.\left|\mathrm{S}_{41}\right|\right)$ are far apart. 


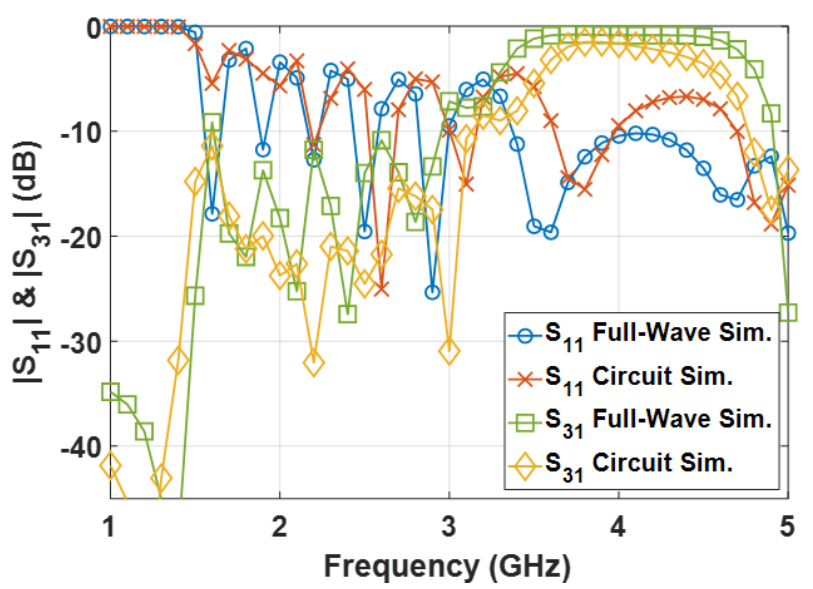

(a)

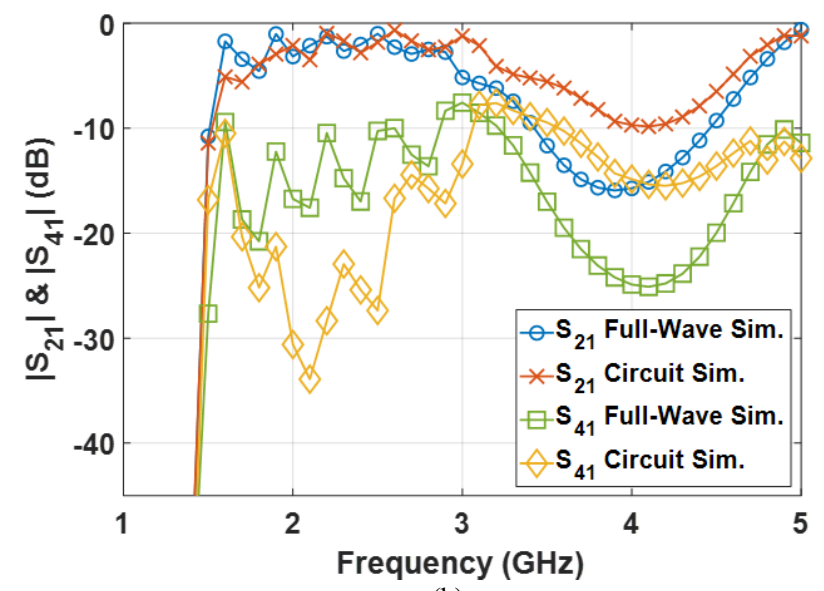

(b)

Fig. 8. S-parameters of the CRLH coupler: (a) $\left|S_{11}\right| \&\left|S_{31}\right|$, (b) $\left|S_{21}\right| \&\left|S_{41}\right|$.

\section{CONCLUSION}

Complete and unambiguous presentation of CRLH TLs is possible only when loss parameters are taken into account. Lossless CRLH TLs can be well described as the limiting case of lossy TLs. Developing an accurate circuit model for both CRLH TLs and couplers is possible without exploiting curve fitting methods and using analytic expressions, solely.

\section{REFERENCES}

[1] C. Caloz and T. Itoh, "Novel microwave devices and structures based on the transmission line approach of meta-materials," in IEEE MTT-S Int. Symp., Philadelphia, PA, June 2003, pp. 195-198.

[2] C. Caloz and T. Itoh. Electromagnetic Metamaterials. Wiley-IEEE Press, 2006.

[3] C. Caloz, A. Sanada, and T. Itoh, "A novel composite right-/left-handed coupled-line directional coupler with arbitrary coupling level and broad bandwidth", IEEE Trans. Microw. Theory Tech., vol. 52, no. 3, pp. 980992, 2004.

[4] F.P. Casares-Miranda, C. Camacho-Penalosa, C. Caloz, "High-gain active composite right/left-handed leaky-wave antenna", IEEE Trans. Antennas Propag., vol. 54, no. 8, pp. 2292-2300, 2006.

[5] S. Mao and M. Wu, "A novel 3-dB directional coupler with broad bandwidth and compact size using composite right/left-handed coplanar waveguides", IEEE Microw. Wirel. Compon. Lett., vol. 17, no. 5, pp. 331333, 2007.

[6] M. Selvanayagam, G.V. Eleftheriades, "Negative-refractive-index transmission lines with expanded unit cells", IEEE Trans. Antennas Propag., vol. 56, no. 11, pp. 3592-3596, 2008.
[7] C. Lai, S. Chiu, H. Li, S. Chen, "Zeroth-Order Resonator Antennas Using Inductor-Loaded and Capacitor-Loaded CPWs", IEEE Trans. Antennas Propag., vol. 59, no. 9, pp. 3448-3453, 2011.

[8] D. Kim, J. Lee, "Beam scanning leaky-wave slot antenna using balanced CRLH waveguide operating above the cutoff frequency", IEEE Trans. Antennas Propag., vol. 61, no. 5, pp. 2432-2440, 2013.

[9] P. Chi and C. Liu, "Novel dual-band quasi-0-dB coupled-line coupler using the composite right/left-handed transmission lines", IEEE Trans Compon Packaging Manuf Technol, vol. 4, no. 2, pp. 259-267, 2014.

[10] Z.L. Ma, L.J. Jiang, S. Gupta, W.E.I. Sha, "Dispersion characteristics analysis of one dimensional multiple periodic structures and their applications to antennas", IEEE Trans. Antennas Propag., vol. 63, no. 1, pp. 113-121, 2015.

[11] Z. Qamar, S.Y. Zheng, W.S. Chan, D. Ho, "An equal-length multiway differential metamaterial phase shifter", IEEE Trans. Microw. Theory Tech., vol. 65, no. 1, pp. 136-146, 2017.

[12] D.K. Karmokar, S. Chen, T.S. Bird, Y.J. Guo, "Single-layer multi-via loaded CRLH leaky-wave antennas for wide-angle beam scanning with consistent gain", IEEE Microw. Wirel. Compon. Lett., vol. 18, no. 2, pp. 313-317, 2019.

[13] L. Szymanski and A. Grbic, "2-D circuit-based bianisotropic omega media," IEEE Trans. Antennas Propag., vol. 68, no. 11, pp. 7395-7405, 2020.

[14] C. Caloz and T. Itoh, "Lossy transmission line metamaterials", Microw. Opt. Technol. Lett., vol. 43, no. 2, pp. 112-114, 2004.

[15] R.F. Harrington. Time-Harmonic Electromagnetic Fields. Wiley-IEEE Press, 2001.

[16] D.M. Pozar. Microwave Engineering. John Wiley \& Sons, Inc., 2012.

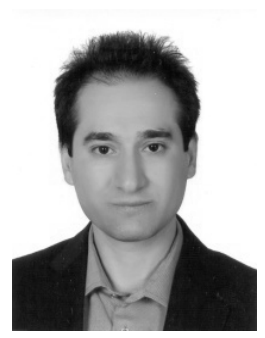

Babak Honarbakhsh was born in Tehran, Iran, in 1981. He received his B.S., M.S., and $\mathrm{Ph} . \mathrm{D}$. degrees in electrical engineering, all from Amirkabir University of Technology (Tehran Polytechnic), in 2004, 2007, and 2012. He is currently an Assistant Professor in the Department of Electrical Engineering at Shahid Beheshti University. His research interest is numerical electromagnetics. 